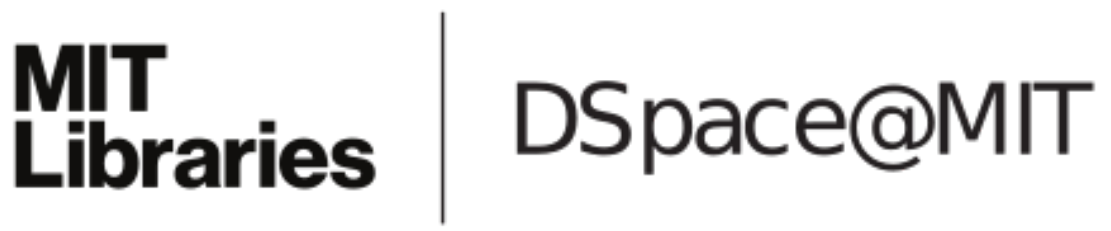

\author{
MIT Open Access Articles
}

Electronic Properties of Disordered Organic
Semiconductors via QM/MM Simulations

The MIT Faculty has made this article openly available. Please share how this access benefits you. Your story matters.

Citation: Difley, Seth et al. "Electronic Properties of Disordered Organic Semiconductors via QM/ MM Simulations." Accounts of Chemical Research 43.7 (2010): 995-1004.

As Published: http://dx.doi.org/10.1021/ar900246s

Publisher: American Chemical Society

Persistent URL: http://hdl.handle.net/1721.1/69632

Version: Author's final manuscript: final author's manuscript post peer review, without publisher's formatting or copy editing

Terms of Use: Article is made available in accordance with the publisher's policy and may be subject to US copyright law. Please refer to the publisher's site for terms of use. 


\title{
Electronic Properties of Disordered Organic Semiconductors via QM/MM Simulations
}

\author{
Seth Difley, Lee-Ping Wang, Sina Yeganeh, Shane R. Yost, and Troy Van Voorhis* \\ Department of Chemistry, Massachusetts Institute of Technology, Cambridge, Massachusetts \\ $02139-4307$ \\ E-mail: tvan@mit.edu
}

\section{Conspectus}

Organic semiconductors (OSCs) have recently received significant attention for their potential use in photovoltaic, LED and FET devices. Part of the appeal of OSCs is their robustness to material disorder (e.g. packing defects), making them more flexible and easier to process than their inorganic counterparts. In addition to their technological applications, OSCs provide an attractive laboratory for examining the chemistry of heterogeneous systems. In particular, because OSCs are both electrically and optically active, one has access to a wealth of electrical and spectroscopic probes that are sensitive to a variety of localized electronic states in these materials. In this tutorial, we review the basic concepts involved in first-principles modeling of the electronic properties of disordered OSCs. There are three theoretical ingredients in the computational recipe. First, Marcus theory of nonadiabatic electron transfer (ET) provides a direct link between energy and kinetics. Second, constrained density functional theory (CDFT) forms the basis for an ab initio model of the diabatic charge states required in ET. Finally, quantum mechanical/molecular mechanical (QM/MM) techniques allow us to incorporate the influence of the heterogeneous environment on the diabatic states. As an illustration,

${ }^{*}$ To whom correspondence should be addressed 
we apply these ideas to the small molecule OSC tris(8-hydroxyquinolinato)aluminum $\left(\mathrm{Alq}_{3}\right)$. In films, $\mathrm{Alq}_{3}$ can possess a large degree of short-range order, placing it in the middle of the order-disorder spectrum - with pure crystals on one end and totally amorphous structures on the other. We show that the QM/MM recipe reproduces the transport gap, charge carrier hopping integrals, optical spectra and reorganization energies of $\mathrm{Alq}_{3}$ in quantitative agreement with available experiments. However, none of these quantities can be specified accurately by a single number. Instead, each property is characterized by a distribution that reflects the influence of the heterogeneous environment on the electronic states involved. For example, the hopping integral between a given pair of $\mathrm{Alq}_{3}$ molecules can vary by as much as a factor of five on the nanosecond timescale, while the integrals for two different pairs can easily differ by a factor of 100. In order to make accurate predictions of mesoscopic properties such as carrier mobilities based on these calculations, one must account for the dynamic range of the microscopic inputs, rather than just their average values. We thus find that many of the computational tools required to characterize these materials are now in hand. As we continue to improve this computational toolbox, one envisions a future scenario in which, given some information about OSC deposition, one could simulate device operation on the atomic scale to obtain data that not only aids in the interpretation of experimental results but also guides the design of more efficient devices.

\section{Introduction}

The study of organic electronics presents a new landscape upon which chemists, materials scientists, and others are working to supplement conventional inorganic semiconductor devices with a palette of small molecule and polymeric materials. ${ }^{1}$ These are typically $\pi$-conjugated systems with low-lying excited states and the ability to transport both holes and electrons. ${ }^{2}$ While work remains to be done, significant progress has been made on device applications such as organic light-emitting diodes (OLEDs),photovoltaics (OPVs), and field-effect transistors (OFETs). ${ }^{3}$

Although monocrystalline organic materials can be produced, most applications are limited by 
cost to polycrystalline or amorphous structures. Thus disorder greatly affects carrier mobilities and generation-recombination behavior of OSC devices. In addition, because there are no intermolecular covalent bonds, weak van der Waals (vdW) forces dominate interactions in the solid ${ }^{3}$ creating localized (Frenkel type) excitons while strong electron-phonon interactions lead to polaron formation and hopping-type transport. The conceptual simplicity of bands in traditional semiconductors is thus replaced by a complex disordered system where electron interactions and electron-phonon coupling determine the energy landscape. ${ }^{2}$

In this picture, the key quantities are the energies and couplings betwee the different localized electronic states, and an array of computational tools exist that give us access this information. Individual chromophores can be described with semi-empirical, ${ }^{4}$ density functional theory (DFT), ${ }^{5,6}$ or wavefunction-based methods. ${ }^{7}$ To describe short-range disorder in the solid, simulated annealing can generate dimer structures for which the electronic coupling between pairs of molecules can be computed, leading to an estimate of carrier mobility. ${ }^{8}$ To crudely address electrostatic effects of the environment, polarizable continuum models (PCMs) can also be invoked. ${ }^{9}$

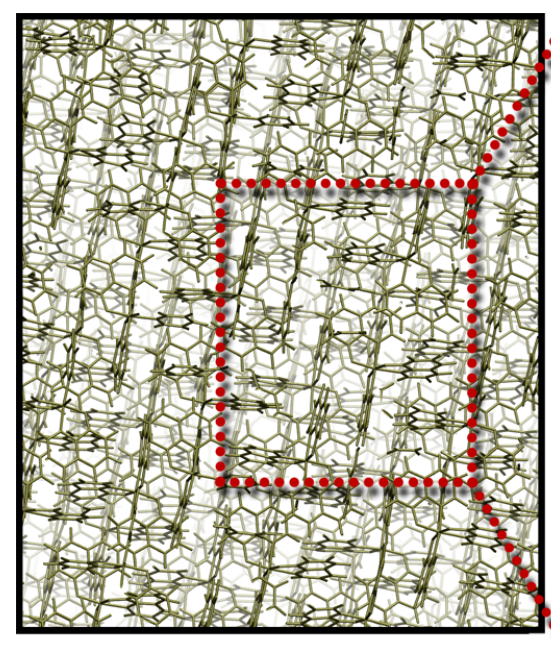

Classical Mechanics

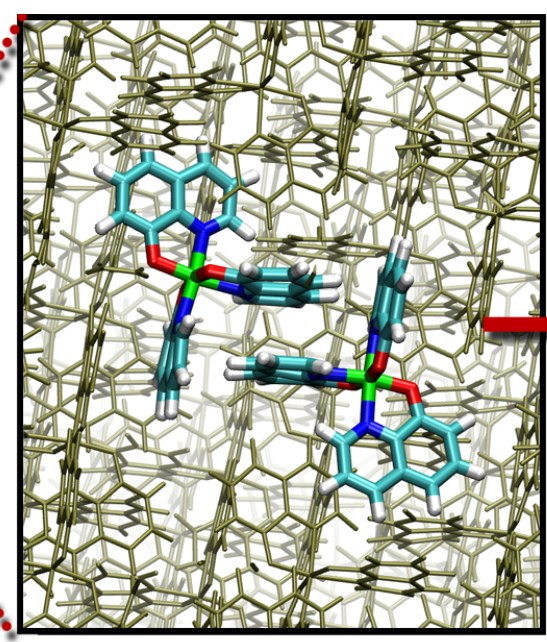

QM/MM Embedding

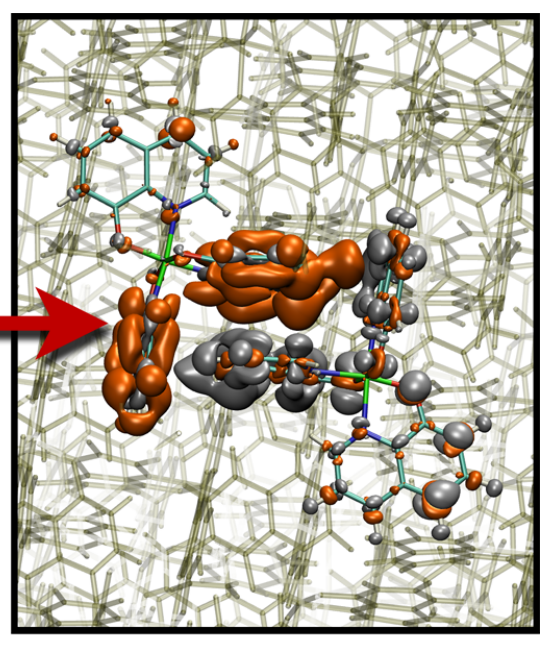

All Electron Calculation

Figure 1: Illustration of QM/MM method. Left: Disordered Alq 3 supercell, generated using MM simulation techniques. Center: Selection of QM region within the MM environment. Right: Electronic structure calculation of desired electronic state.

QM/MM studies ${ }^{10}$ can shed more light on the impact of the environment on the energy landscape. By explicitly including intermolecular interactions in the QM/MM potential, the effect of 
neighboring molecules on optical and transport properties can be better described. Even for an amorphous material with nanometer-scale disorder, the localized nature of the electronic states means that the QM region need only include one or two monomers (See Figure 1). This makes QM/MM calculations vastly faster than a full DFT calculation on the same system.

Recently, we and others ${ }^{8,11}$ have begun a systematic study of organic materials with QM/MM methods. This review is a tutorial intended to illustrate the fundamental principles involved in this new class of simulations. We begin by introducing the relevant theoretical ingredients - the Marcus picture of ET, identifying diabatic states using CDFT, and the integration with QM/MM techniques. To illustrate how the techniques interface with one another, we focus on $\mathrm{Alq}_{3}$, a well-studied

molecule $\mathrm{e}^{8,9,12-14}$ that is the prototypical OLED material.We apply the QM/MM methodology to $\mathrm{Alq}_{3}$ and compute a range of electronic properties: transport gap, electron- and hole- transfer rates, spectral densities and charge carrier recombination rates. At every stage, we observe the crucial influence of the environment, and particularly disorder, on the distribution of electronic states. We conclude by summarizing the strengths and weaknesses of this approach for studying OSCs and detail future work necessary for broader applications.

\section{Theory}

\subsection{Marcus Theory of Electron Transfer}

In the condensed phase, ET occurs as the static and dynamic polarization of the environment stabilizes (or destabilizes) charged species. For nonadiabatic reactions (see Figure 2) the landscape is described by two parameters: the reorganization energy, $\lambda$, which describes the relaxation of the entire system as a result of the new electronic state, and $\Delta G$, the free energy change attending ET. The intramolecular ET rate, $k_{E T}$, can be aproximately written as ${ }^{15}$

$$
k_{E T}=\frac{2 \pi}{\hbar}\left|V_{R P}\right|^{2} \frac{1}{\sqrt{4 \pi \lambda k_{B} T}} \exp \left(\frac{-(\lambda+\Delta G)^{2}}{4 \lambda k_{B} T}\right) .
$$


Here, the prefactor involves the electronic coupling element, $V_{R P}$, between the (diabatic) reactant and product states of ET.

In solid state systems, the reactant and product states are the polarons that determine charge transport. ${ }^{16} \lambda$ leads to polaron trapping via two mechanisms: the relaxation of the "inner sphere" molecules undergoing ET $\left(\lambda_{i}\right)$, and the polarization of the "outer sphere" molecules in the en-

a) $\mathrm{R}$

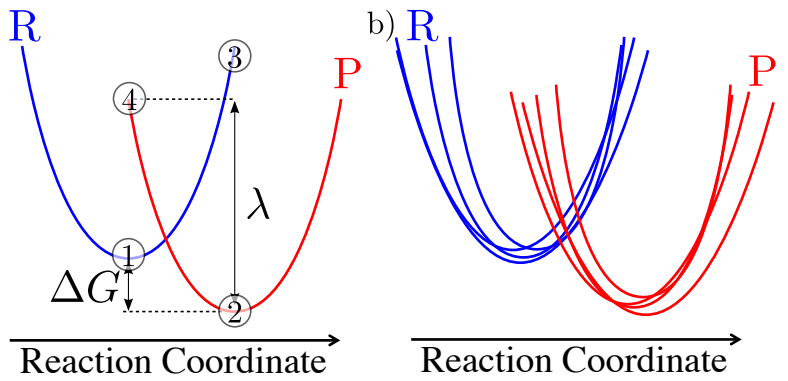

Figure 2: Marcus free energies describing a transition from reactant $(\mathrm{R})$ to product $(\mathrm{P})$ states. a) Circles indicate calculations for four-point determination of $\lambda, \Delta G$. b) Static disorder creates a distribution of reactant and product energy landscapes. vironment $\left(\lambda_{o}\right)$. Meanwhile, charge hopping is generated by the tunneling matrix element $V_{R P} \equiv\langle P|\hat{H}| R\rangle$, where $|R\rangle(|P\rangle)$ is the reactant (product) state, and $\hat{H}$ is the electronic Hamiltonian. $V_{R P}$ is often assumed constant along the reaction coordinate (Condon approximation). While this turns out to be a good approximation for $\mathrm{Alq}_{3}$ (there is very little correlation between $\Delta G$ and $V_{R P}$ ) in general we avoid this approximation and instead compute $V_{R P}$ at each instantaneous configuration.

We note that Marcus theory is typically an ensemble theory. A given reaction is characterized by a single value of $\Delta G, \lambda$, and $V_{R P}$ (Figure 2a). Meanwhile, organic solids tend to be disordered, nonequilibrium systems. As a result, an OSC has many relevant values of $\Delta G, \lambda$, and $V_{R P}$ (Figure $2 b$ ) leading to a distribution of ET rates via Eq. 1. In order to translate the tangle of energy landscapes in Figure $2 \mathrm{~b}$ into a single rate, one must resolve the individual timescales of relaxation versus charge hopping. In what follows, we will not unravel this particular Gordian knot but instead focus on quantifying the underlying distributions.

\subsection{Constrained DFT as a Route to Diabatic States}

In order to connect the Marcus picture to a physical system, a prescription for diabatic electronic states must be chosen. In this vein, empirical valence bond, ${ }^{10}$ semiempirical molecular orbital, ${ }^{4} \mathrm{CDFT}^{17,18}$ and CI-based ${ }^{19}$ approaches have all proven useful. In what follows, we em- 
ploy CDFT to define diabatic electronic states and compute relevant ET parameters. We choose this route because CDFT provides a compromise between computational cost and accuracy: the calculations are fast enough for realistic systems but also reliable enough for chemical accuracy in many properties.

Within CDFT, a diabatic ET state such as $D^{+} A^{-}$is defined to be the lowest energy state such that the partial charges on the atoms that compose the acceptor $(A)$ sum to -1 . If the overall system is neutral, the donor $(D)$ atom charges will then sum to +1 , reflecting the hole left behind by the transferred electron. Other diabatic states, such as $A A^{-}$or $D^{+} D$ are defined analogously. The constraint is enforced by applying a local chemical potential bias, $V$, between donor and acceptor. By varying the strength of this potential, one can control the charge on the acceptor. In CDFT, $V$ is determined self-consistently with the charge density to determine the lowest energy state that satisfies the charge constraint exactly. ${ }^{20}$ The charge-constrained states thus obtained form the foundation for a seamless connection between DFT and ET theory. ${ }^{17,21}$

For a rigid system like an organic crystal, the entropic contributions to the Marcus parameters $(\Delta G, \lambda)$ are expected to be small, so the task reduces to computing enthalpies. These enthalpies can be computed from a combination of geometry optimizations and energy evaluations. Adopting a notation of $(X \mid Y)$ for the energy of state $X$ at the optimized geometry of $Y$, we can compute the driving force and reorganization of $R \rightarrow P$ from (see Figure 2)

$$
\begin{aligned}
\Delta G & \equiv \frac{1}{2}[(R \mid R)+(R \mid P)-(P \mid P)-(P \mid R)] \\
\lambda & \equiv-\frac{1}{2}[(R \mid R)-(R \mid P)+(P \mid P)-(P \mid R)]
\end{aligned}
$$

These expressions make the standard linear response assumption that both free energy surfaces are perfect parabolas with the same curvature. ${ }^{15}$ Finally, we compute $V_{R P}$ in CDFT from a combination of the overlap between electronic states and the matrix elements of the chemical potential between them: ${ }^{22}$

$$
V_{R P}=\left\langle\Psi_{R}|\hat{H}| \Psi_{P}\right\rangle=\frac{F_{R}+F_{P}}{2}\left\langle\Psi_{R} \mid \Psi_{P}\right\rangle-\frac{1}{2}\left\langle\Psi_{R}\left|V_{R}+V_{P}\right| \Psi_{P}\right\rangle
$$


where $F_{R}, V_{R}\left(F_{P}, V_{P}\right)$ are the free energy and constraining chemical potential of the reactant (product) state. Recent applications have shown that CDFT can provide accurate predictions of $\Delta G, \lambda$, and $V_{R P}$ when used within this framework. ${ }^{17,18,21}$

\subsection{QM/MM Modeling of Disordered Systems}

QM/MM techniques have a long history of application to systems where a small active site is surrounded by a much larger environment. The simulation cell is thus divided into classical (MM) and quantum $(\mathrm{QM})$ regions, which interact via the Hamiltonian. ${ }^{10}$ The important quantum phenomena are contained within the molecules of the QM region, while the surrounding MM region provides essential corrections (e.g. local electric fields) from the heterogeneous environment.

The total energy of the QM/MM simulation is given by:

$$
E=E_{\mathrm{QM}}\left(\mathbf{r}_{\mathrm{Q}}\right)+E_{\mathrm{MM}}\left(\mathbf{r}_{\mathrm{M}}\right)+E_{\mathrm{QM} / \mathrm{MM}}\left(\mathbf{r}_{\mathrm{Q}}, \mathbf{r}_{\mathrm{M}}\right) .
$$

Here, $E_{\mathrm{QM}}$ and $E_{\mathrm{MM}}$ are the energies of the QM and MM regions, which depend on the coordinates of the $\mathrm{QM}\left(\mathbf{r}_{\mathrm{Q}}\right)$ and $\mathrm{MM}\left(\mathbf{r}_{\mathrm{M}}\right)$ nuclei respectively. $E_{\mathrm{QM} / \mathrm{MM}}$ represents the interaction energy between the regions and consists of electrostatic interactions plus an empirical vdW repulsion:

$$
E_{\mathrm{QM} / \mathrm{MM}}=-\int \sum_{i \in \mathrm{M}} \frac{q_{i}}{\left|\mathbf{r}_{i}-\mathbf{r}^{\prime}\right|} \rho\left(\mathbf{r}^{\prime}\right) d \mathbf{r}^{\prime}+\sum_{i \in \mathrm{M}} \sum_{j \in \mathrm{Q}} \frac{q_{i} Z_{j}}{\left|\mathbf{r}_{i}-\mathbf{r}_{j}\right|}+\sum_{i \in \mathrm{M}} \sum_{j \in \mathrm{Q}} V_{\mathrm{vdW}}\left(\mathbf{r}_{i}, \mathbf{r}_{j}\right)
$$

In addition to simple electrostatic effects, the QM/MM treatment must account for the fact that the OSC environment is polarizable. We do this by introducing fictitous "Drude" charges, $q_{i}^{\mathrm{d}}$, which are harmonically bound to MM atoms: ${ }^{23}$

$$
E_{\text {Drude }}=\sum_{i} \frac{1}{2} k_{i}^{\mathrm{d}}\left|\mathbf{d}_{i}-\mathbf{r}_{i}\right|^{2}+\sum_{i, j} \frac{q_{i}^{\mathrm{d}} q_{j}}{\left|\mathbf{d}_{i}-\mathbf{r}_{j}\right|}+\sum_{i, j} \frac{q_{i}^{\mathrm{d}} q_{j}^{\mathrm{d}}}{\left|\mathbf{d}_{i}-\mathbf{d}_{j}\right|}
$$

Electronic polarization is modeled by allowing the Drude particles to respond instantaneously to local electric fields, so that for any given nuclear configuration $\{\mathbf{r}\}, E_{\text {Drude }}$ is minimized with 
respect to the positions of the Drude particles. The positions of the Drude particles must be determined self-consistently with the QM charge density, because the electric field experienced by the Drude particles depends on the QM charges and vice versa. For $\mathrm{Alq}_{3}$, the empirical parameters contained in the FF terms above were fitted to reproduce available experimental and ab initio data as detailed in the supporting information.

\subsection{Simulation Procedure}

We begin our QM/MM calculations by obtaining thermally sampled configurations of the OSC material. A pure MM trajectory with a large number of molecules is simulated at constant temperature, from which we extract a large number of snapshots at fixed time intervals for QM/MM calculations.

Each snapshot contains a disordered supercell of many molecules (Figure 1, left pane). Because electronic states in OSCs are largely localized, molecules distant from the active region mostly contribute long-range effects that are well described by MM. We invoke QM/MM by selecting a small subset of the snapshot (Figure 1, center pane), which by physical intuition we assume to contain the interesting quantum effects. The quantum region is intentionally chosen as small as possible to minimize the spurrious charge delocalization problems that plague DFT. ${ }^{17}$ Finally, the QM molecules are embedded in the MM simulation and treated using a full QM electronic structure calculation (Figure 1, right pane); the interaction with the MM system is replaced by the QM/MM Hamiltonian (Eq. 5). The MM atomic charges appear as external point charges in the QM calculation, which affect the QM electronic density.

At the boundary between the regions, QM electron density can localize unphysically around nearby MM point charges (i.e. the system transfers charge to the environment). We can repair this by replacing MM point charges with Gaussians, $\rho(r)=\frac{1}{\sigma \sqrt{2 \pi}} e^{-\frac{\left(r-r_{0}\right)^{2}}{2 \sigma^{2}}}$. In this work, we choose the blurring parameter $\sigma=3.5 \AA$, approximately half the radius of $\mathrm{Alq}_{3}$. Finally, forces on QM and MM atoms alike are given by differentiating the QM/MM Hamiltonian with respect to the nuclear coordinates. When an optimized structure is desired (e.g. when computing $\lambda$ ) these forces are 
used to minimize the energy of the entire system.

\section{Computational Details}

\section{1 $\quad \mathrm{Alq}_{3}$ as a Model Material}

$\mathrm{Alq}_{3}$ is an octahedral coordination complex in which three 8-hydroxyquinoline ligands are bound to a central aluminum atom. Numerous experimental and theoretical studies are available in the literature, ${ }^{8,9,12,13,24,25}$ making this material an ideal candidate for illustrating the methodology. $\mathrm{Alq}_{3}$ is known to exist in several different crystalline phases; the experimentally determined ${ }^{26}$ unit cell of $\beta-\mathrm{Alq}_{3}$ is used here as our initial structure because it most closely resembles that of the amorphous film. ${ }^{27}$ We constructed a triclinic supercell of $6 \times 6 \times 6 \mathrm{Alq}_{3}$ unit cells $(\mathrm{Z}=2)$ containing 432 molecules. For consistency with our QM method, our simulation cell used the equilibrium monomer geometry from a gas-phase B3LYP/3-21G geometry optimization, placed in maximal coincidence with the experimental molecular coordinates (RMSD $<0.1 \AA$ ).

\subsection{Sampling and QM/MM Methodology}

We performed non-polarizable MM sampling in GROMACS $4.0^{28}$ using the NVT ensemble at 300 K. Full periodic boundary conditions were applied, and bond lengths were held to DFT-optimized values using the LINCS algorithm. The entire simulation length was 5.0ns, intended to sample typical thermal fluctuations in this material. 500ps was allowed for thermal equilibration, after which snapshots were extracted at 20ps intervals to minimize shot-to-shot correlation. QM/MM calculations were performed with the CHARMM ${ }^{29}$-Q-Chem ${ }^{30}$ interface. ${ }^{31}$ Where noted, the QM/MM calculations account for electronic polarizability of the MM system through the presence of the Drude particles. 


\subsection{Electronic Structure Calculations}

Electronic structure calculations were all performed using the 3-21G basis set at the B3LYP level of theory. To investigate the basis set dependence of each property, we performed selected calculations using the larger cc-pVDZ and aug-cc-pVDZ basis sets. Unless specifically noted, we find negligible basis set dependence ( $<5 \%$ change) for all of the properties discussed in the text.

We determine most excitation energies using time-dependent DFT (TDDFT), ${ }^{32}$ with the exception of CT states, which are obtained with CDFT. Becke weights are used to define electron populations in the CDFT framework with charge and spin constrained together.

\section{Charge Transport Properties}

The motion of charge carriers in OSCs is governed by several material properties. First, the transport gap (also called the band gap) measures the energy difference between free electron and hole carriers. It is formally given by the difference between the ionization potential (IP) and electron affinity (EA), $E_{t}=\mathrm{IP}-\mathrm{EA}$. In OSCs the gap is modulated both by the heterogeneity of the system (which gives rise to a distribution of local IPs and EAs) and the intrinsic band width, which is governed by the electronic coupling $V_{R P}$ (also called the hopping integral). Meanwhile, the carrier mobility $\left(\mu_{e, h}\right)$ is determined by the Marcus ET rates, which is in turn are influenced both by energetic disorder and the magnitude of $V_{R P}{ }^{2,5}$

\subsection{Transport Gap}

The prescription for computing the transport gap in $\mathrm{Alq}_{3}$ is straightforward. For a given snapshot, we choose a single monomer and compute QM/MM energies for the cation, anion, and neutral states yielding the transport gap, $E_{t}=E_{+}+$



Figyre 3: $\mathrm{Alq}_{3}$ electron affinity (EA) and ionization potential (IP) computed with fixed MM point charges (thin solid curves), with only charge blurring (dashed curves), and with only polarization correction (thick solid curves). The transport gap, $E_{t}$, is the difference between the IP and EA distribution onsets. All 
$E_{-}-2 E_{0}$. By computing $E_{t}$ for different

monomers at a variety of snapshots, we can obtain an estimate of the variations in the gap due to disorder.

In Figure 3, simulation results for EAs and IPs are presented. At a crude level, we first compute the gap with fixed MM point charges, and obtain an EA (IP) of $0.6 \pm 0.05(6.3 \pm 0.06) \mathrm{eV}$ and a transport gap of $5.4 \mathrm{eV}$ (as measured from the IP and EA onsets). Blurring of the MM charges blue-shifts both EA and IP by $.15 \mathrm{eV}$ yielding no effect on $E_{t}$. Including polarization corrections in the FF blue-shifts the EA onset to $1.3 \pm 0.05 \mathrm{eV}$ and red-shifts the IP onset to $5.7 \pm 0.05 \mathrm{eV}$ to give an edge-to-edge transport gap of $4.3 \mathrm{eV}$. This $1.3 \mathrm{eV}$ polarization correction is a result of the strong interactions of the anion and cation with the charged Drude particles. Indeed, assuming each charge sits in a sphere of radius $.7 \mathrm{~nm}$ surrounded by a dielectric medium with $\varepsilon_{s}=2.9$, we obtain $\Delta E_{p o l}=0.68 \mathrm{eV}$ for each charge - very close to the observed polarization shift of $0.55 \mathrm{eV}(0.65 \mathrm{eV})$ for the IP (EA). A small representative set of calculations performed using the aug-cc-pVDZ basis red-shifts the $E_{t}$ modestly by $0.2 \mathrm{eV}$. Combining the basis set and polarization corrections gives a corrected estimate of $4.1 \mathrm{eV}$ for the transport gap. Thus we see that disorder has a very small effect on the transport levels of $\mathrm{Alq}_{3}$ : the shot-to-shot variation in IP or EA is only $0.05 \mathrm{eV}$, and the site to site variation (not shown) is only $0.01-0.02 \mathrm{eV}$.

A variety of experimental and theoretical results are available for comparison. Experimentally, IPs and EAs of $\mathrm{Alq}_{3}$ have been determined, yielding band gaps in the range $3.6-4.1 \mathrm{eV} .{ }^{24,25,33}$ Our result is at the higher edge of this range. Previous theoretical studies on $\mathrm{Alq}_{3}$ monomers within a PCM report an EA (IP) of $1.7 \mathrm{eV}(5.8 \mathrm{eV})^{9}$ yielding a transport gap of $4.2 \mathrm{eV}$. Our results are in excellent agreement with these predictions. Although the less expensive PCM calculations agree well with our results, they can only give average values of EA/IP, while the QM/MM treatment also yields distributions describing the effect of disorder on the energetics in OSCs. 


\subsection{Electronic Coupling}

To obtain electronic couplings, we compute the matrix elements $\left(A \equiv \mathrm{Alq}_{3}\right)\left\langle A^{-} A|\hat{H}| A A^{-}\right\rangle$(for electron transport) and $\left\langle A^{+} A|\hat{H}| A A^{+}\right\rangle$(for hole transport). We take the quantum region to be a pair of $\mathrm{Alq}_{3}$ molecules with an excess electron or hole. Two CDFT states are constructed in which the excess charge is constrained to be on one molecule or the other and the electronic coupling between these states is computed using Eq. 4. This is repeated for several near-neighbor pairs at a large number of snapshots. While nuclear heterogeneity has little effect on electron and hole energies, it is expected to significantly influence the electron and hole couplings because of their strong dependence on relative orientation.

Figure 4 shows the B3LYP/3-21G electron and hole hopping parameters for several different pairs (indexed by the lattice vector separating them) with fixed MM point charges. The inset shows that while charge blurring can change individual couplings up to $100 \mathrm{meV}$, the distributions are not significantly shifted. For a given dimer we note the coupling can vary

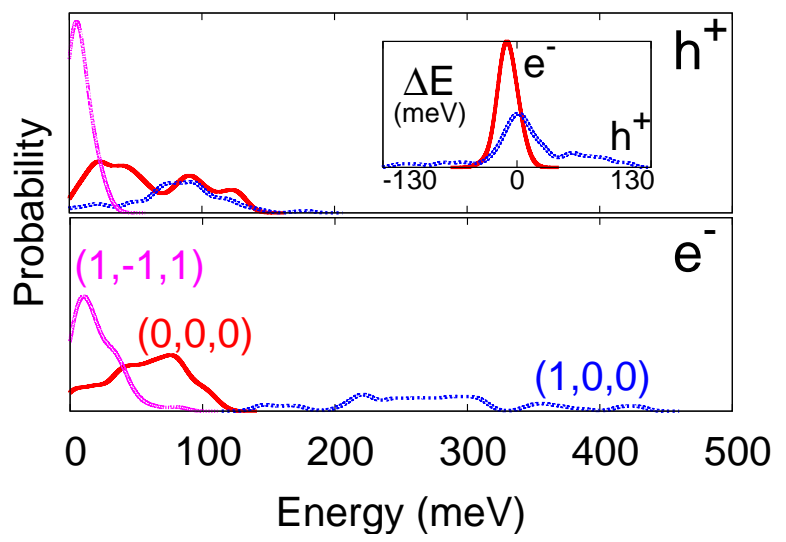

Figure 4: Electron $\left(\mathrm{e}^{-}\right)$and hole $\left(\mathrm{h}^{+}\right)$electronic coupling distributions. Inset: The difference, $\Delta E=E_{\text {blur }}-E_{\text {noblur }}$, between blurred and unblurred couplings.

by as much as a factor of five as a function of time - suggesting significant dynamic heterogeneity of the hopping rate on the nanosecond timescale. There is also obvious static structure in the data: despite the width of the distributions, the couplings typically obey $V^{h}(1,-1,1)<V^{h}(1,0,0) \approx$ $V^{h}(0,0,0)$ and $V^{e}(1,-1,1)<V^{e}(0,0,0)<V^{e}(1,0,0)$. Finally, we note that holes tend to have a higher density of very small couplings, while electrons account for most of the high couplings.

Previous theoretical efforts have shown that electron couplings in the perfect $\beta$ crystal can be up to $289 \mathrm{meV}$ - ten times as large as the largest hole coupling. ${ }^{27}$ Meanwhile, in an amorphous sample both electron and hole couplings are in the $0-100 \mathrm{meV}$ range. ${ }^{8}$ As our simulations are on a thermalized crystal structure, lying somewhere between the two aforementioned systems, we see 
that disorder tends to progressively enhance small hole couplings while disrupting some of the strong electron transport pathways in $\mathrm{Alq}_{3}$.

\subsection{Reorganization and Mobility}

$\lambda^{e}\left(\lambda^{h}\right)$ can be computed by: 1) constraining the electron (hole) to be on one monomer and relaxing the structure of the whole system, 2) repeating the process with the electron (hole) constrained on the opposite monomer, and 3) computing $\lambda$ as in Eq. 3. This process takes tens to hundreds of times longer than an energy calculation, and so it is not computationally feasible to compute $\lambda$ for every pair of monomers or every snapshot. In order to generate Table 1, we have therefore modeled only a handful of dimers from a single snapshot.

Table 1: Calculated Marcus parameters and rates for various donor/acceptor pairs within Alq 3 crystal, labelled by the lattice vector between the monomers. All energies are reported in meV except for $\Delta \mathrm{G}^{C T}$ (eV). Rates are reported in $\mathrm{s}^{-1}$. Rows are arranged in order of increasing Al-Al separation.

\begin{tabular}{|c|c|c|c|c|c|c|c|c|c|c|c|}
\hline \multirow{2}{*}{$\begin{array}{c}\text { Lattice } \\
\text { Vector }\end{array}$} & \multicolumn{4}{|c|}{ Hole Transport } & \multicolumn{4}{c|}{ Electron Transport } & \multicolumn{3}{c|}{ Recombination } \\
\cline { 2 - 13 } & $\lambda^{h}$ & $\Delta G^{h}$ & $V^{h}$ & $\log _{10}\left(k_{h}\right)$ & $\lambda^{e}$ & $\Delta G^{e}$ & $V^{e}$ & $\log _{10}\left(k_{e}\right)$ & $\lambda^{C T}$ & $\Delta G^{C T}$ & $V_{R P}$ \\
\hline$(1,0,1)$ & 333 & -38 & 87 & 14.0 & 352 & 25 & 115 & 14.0 & 653 & -3.07 & 104 \\
\hline$(0,-1,0)$ & 245 & 36 & 45 & 13.4 & 285 & -6 & 45 & 13.4 & 498 & -3.26 & 69 \\
\hline$(0,0,1)$ & 272 & -30 & 87 & 14.1 & 377 & 38 & 63 & 13.4 & 337 & -3.38 & 119 \\
\hline$( \pm 1,0,0)$ & 245 & -39 & 46 & 13.6 & 366 & -171 & 33 & 13.4 & 614 & -3.08 & 86 \\
\hline$(0,0,0)$ & 162 & -47 & 35 & 13.6 & 238 & -18 & 86 & 14.1 & 457 & -3.20 & 195 \\
\hline$(1,-1,0)$ & 272 & 16 & 20 & 12.7 & 492 & 9 & 52 & 13.1 & 532 & -2.98 & 196 \\
\hline$(1,0,0)$ & 220 & -6 & 94 & 14.2 & 253 & -17 & 317 & 15.2 & 283 & -3.50 & 159 \\
\hline$(0, \pm 1,0)$ & 210 & -86 & 13 & 12.7 & 338 & 10 & 80 & 13.8 & 364 & -3.54 & 134 \\
\hline$(1,-1,1)$ & 157 & 79 & 3 & 11.3 & 354 & -4 & 4 & 11.2 & 225 & -3.64 & 12 \\
\hline$(0,-1,1)$ & 173 & -3 & $<1$ & 10.3 & 373 & -10 & 3 & 11.1 & 244 & -3.71 & N/A \\
\hline$(-1,-1,0)$ & 173 & -45 & $<1$ & 9.9 & 337 & -62 & 1 & 10.3 & 279 & -3.75 & N/A \\
\hline
\end{tabular}

As shown in Table 1, the typical electron (hole) reorganization energy in $\mathrm{Alq}_{3}$ is $337 \pm 63$ $\mathrm{meV}(223 \pm 47 \mathrm{meV})$. These values are significantly larger than the typical $V_{R P}$, consistent with the generally poor transport properties (and high reorganization barriers) of organic materials. Meanwhile, the distribution of $\Delta G( \pm 48 \mathrm{meV})$ is consistent with the width of the IP and EA distributions in Figure 3. 
We can further estimate $\lambda_{i}$ by performing a gas-phase calculation to find $\lambda_{i}^{e}=216 \mathrm{meV}$ and $\lambda_{i}^{h}=208 \mathrm{meV}$ so that $\lambda_{o}^{e} \approx 111 \mathrm{meV}$ while $\lambda_{o}^{h} \approx 15 \mathrm{meV}$. Thus, the reorganization energy for electrons is larger primarily because the environment traps an excess electron more strongly than an excess hole. This effect can be rationalized by noting that the electron wavefunction is typically more delocalized and therefore more susceptible to environmental fluctuations.

As illustrated in Table 1, with $\lambda, \Delta G$, and $V_{R P}$ for a given pair, we can predict the rate of ET via Eq. 1, and we note that in 8 of 11 cases the rate of ET is larger than that for hole transfer. The primary differentiating factor is that the typical hopping integral for electrons is considerably larger than for holes. In most cases, this is enough to overcome the difference in activation barriers, which typically favors holes. Our finding agrees qualitatively with the experimental observation that $\mathrm{Alq}_{3}$ is a good electron transport material, with $\mu_{e} \approx 100 \mu_{h}$. Because of heterogeneity in $k_{e}$ and $k_{h}$, it is not a trivial matter to make a quantitative prediction of the mobility based on these rates. Since $\mu$ is most sensitive to the slow, rate limiting values of $k$, one must simulate carrier diffusion using the given hopping rates to obtain mobilities. ${ }^{8}$ Such a simulation is beyond the scope of this tutorial, but the feasibility of such mesoscopic simulations provides the final link between atomistic data and bulk properties.

\section{Optical Properties}

The optical response of $\mathrm{Alq}_{3}$ is dominated by two Frenkel-type excitons: a singlet state that fluoresces in the green and a triplet that phosphoresces in the red. However, the activity of $\mathrm{Alq}_{3}$ in OLEDs also depends critically on the distribution of CT excitons, which are intermediates in the process of electron-hole recombination. In contrast with transport properties that describe ground state behavior, optical properties pertain to excited states making them somewhat more challenging from a simulation perspective. In what follows, we focus on just two features: the spectral densities of the various states and the rate of CT recombination to the ground state. 


\subsection{Spectral Density}

To generate singlet and triplet exciton spectral densities, the quantum region of the $\mathrm{QM} / \mathrm{MM}$ system was chosen to be a single monomer, and TDDFT was used to obtain the lowest lying singlet and triplet states at a variety of snapshots. For CT excitons, a $(0,0,0)$ dimer pair was chosen as the quantum region and intermolecular $\mathrm{CT}$ energies were computed as the difference between the $\mathrm{CDFT} A^{+} A^{-}$and $A A$ state energies for each geometry snapshot. The

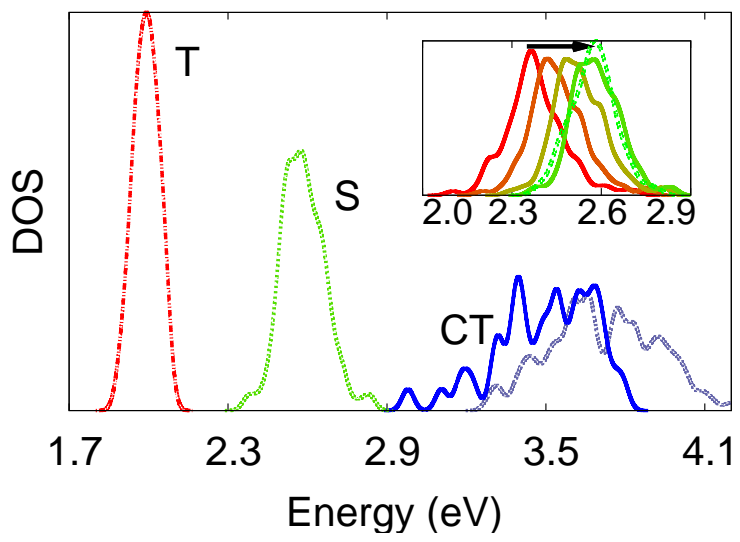

Figure 5: Triplet (T), singlet (S), and CT exciton spectral densities. Solid CT curve includes polarization correction. Inset: Dependence of singlet exciton states on blurring. The arrow indicates increasing $\sigma$, from 0 (no blur) to $\infty$ (no charges). The other states are insensitive to $\sigma$

resulting spectral densities are presented in Figure 5.

Figure 5 shows singlet excitons centered at $2.6 \pm 0.08 \mathrm{eV}$, triplet excitons at $2.0 \pm 0.04 \mathrm{eV}$ and CT state energies at $3.7 \pm 0.2 \mathrm{eV}$ (all with charge blurring). Thus we see immediately that exciton and $\mathrm{CT}$ energies are more sensitive to disorder than either IP or EA. As a result, diffusion of these states will be more strongly influenced by energetic trapping than was the case for charge transport, where fluctuations in the hopping parameter dominated the mobility. The singlet and triplet exciton results can be compared to a range of experimental and theoretical studies. Optical absorbance studies place the singlet exciton at $2.7 \mathrm{eV},{ }^{34}$ while a gas-phase TDDFT calculation finds an exciton energy of $2.8 \mathrm{eV}^{35}$ For the triplet, phosphorescence studies find energies of $2.03-2.10 \mathrm{eV}^{36-38}$ while gas-phase calculations report $2.1 \mathrm{eV}$. Our exciton energies are thus fairly consistent with the existing data and suggest only a very small environmental effect on the Frenkel-type exciton energies.

One important point is that while triplet and CT excitons are insensitive to blurring (as all properties discussed so far have been) the singlet is quite sensitive to blurring (Figure 5, inset). The average singlet energy shifts by $0.3 \mathrm{eV}$ as $\sigma$ is increased from zero (point MM charges) to infinity 
(no MM charges). For the value of $\sigma=3.5 \AA$ used here, there is only a small $(<0.1 \mathrm{eV})$ difference between the QM/MM and pure QM calculations for the singlet, which is physically realistic.

Little is known experimentally about the CT states, so that calculations like the ones presented here are the primary source of information about these intermediates. Figure 5 includes the distribution of CT energies both with and without polarization; polarization shifts the average CT energy by $0.2 \mathrm{eV}$ and clearly increases the density of deeply trapped CT states (i.e. states $>1 \mathrm{eV}$ below the band edge). These deeply trapped states could play an important role in OLEDs as the penultimate state of the electron-hole pair.

\subsection{Electronic Coupling}

To predict the electronic coupling between the neutral ground state and CT exciton, we need to compute the matrix element $\left\langle A^{+} A^{-}|\hat{H}| A A\right\rangle$ using Eq. 4. As previously, we model heterogeneity by computing $V_{R P}$ for multiple geometry snapshots

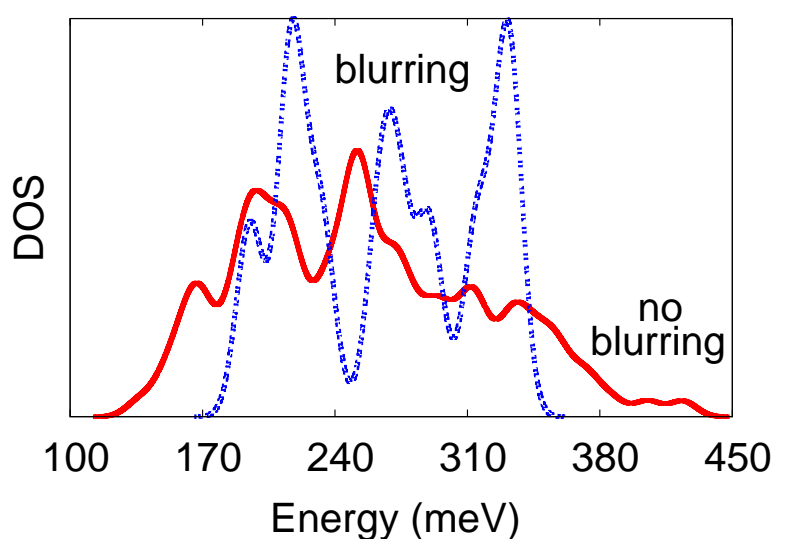
sampled from the MM trajectory. Figure 6 shows the resulting CT electronic Figure 6: CT-ground state state electronic coupling densities. The dashed (solid) density curve is computed with (without) MM charge blurring. coupling for the $(0,0,0)$ dimer using $3-21 \mathrm{G}$. While the distribution is broad, we note that these CT couplings are 2-3 times larger than the typical electron and hole couplings in Figure 4, reflecting the fact that in the CT state the electron and hole attract one another, leading to larger overlap.

We consider two corrections to the results in Figure 6. We see that blurring somewhat narrows, but does not shift, the distribution. Further, applying the polarizable FF is expected to have only a small effect on $V_{R P}$, which primarily involves the reactant and product wavefunctions, not their energies. Thus our results suggest that the CT-to-ground coupling is significantly larger than the electron or hole coupling, tending to increase the charge recombination rate. 


\subsection{Charge-Transfer Reorganization}

Just as for charge transport, there is a $\lambda$ associated with the formation of a localized CT state. However, while charge transport typically occurs in the normal regime $(\Delta G<\lambda)$, the inverted regime $(\Delta G>\lambda)$ is relevant for $\mathrm{CT}$ states. Thus, while small values of $\lambda$ speed up transport, they slow charge recombination. To evaluate the significance of this, we compute the CT reorganization energy for a number of snapshots using Eq. 3 with $R=A^{+} A^{-}$and $P=A A$ (Table 1).

We find $\lambda^{C T}=384 \pm 133 \mathrm{meV}$. If the monomers did not interact, we would expect $\lambda^{C T}$ to be the average of electron and hole reorganization energies: $\frac{1}{2}\left(\lambda^{e}+\lambda^{h}\right)=315 \mathrm{meV}$. However, the dimers here are nearest-neighbors so that a significant portion of the relaxation energy comes from the two monomers approaching each other in the relaxed $A^{+} A^{-}$structure, improving the attractive Coulomb interaction and increasing $\lambda_{i}^{C T}$ relative to the isolated monomers' value. We find that the pairs with the largest $\lambda^{C T}$ also tend to have the smallest Al-Al separations, further supporting this conclusion.

Finally, with the Marcus parameters in hand for several pairs, we can predict the rate of charge recombination to the ground state $\left(k_{c r}\right)$ using Eq. 1 . The results in Table 1 yield extremely slow rates, with a typical CT lifetime of $\gg 1 \mathrm{~s}$. This long lifetime is primarily due to the large disparity between $\Delta G(\approx 3.5 \mathrm{eV})$ and $\lambda^{C T}(\approx .4 \mathrm{eV})$ leading to extremely slow rates in the inverted regime. Thus, direct charge recombination to the ground state is not likely to be a significant loss mechanism for $\mathrm{Alq}_{3}$ in OLED applications, where the rate of exciton formation will be many orders of magnitude faster than these recombination rates.

\section{Conclusions}

In this tutorial, we have demonstrated how simulation can be used as a tool to study disordered OSCs. Taking $\mathrm{Alq}_{3}$ as a prototypical example, we have shown how to compute its electronic properties within a complex, heterogeneous environment. Our results are in good agreement with existing experimental findings, and reinforce the idea that while exciton transport in $\mathrm{Alq}_{3}$ may be 
limited by energetic disorder, charge transport is limited by fluctuations in electronic coupling.

The basis of our investigation is a synthesis of tools that together provide an efficient means of computing accurate electronic properties in a large, disordered system. The primary approximation is the assumption that the electronic states are localized, which is a necessary component for the QM/MM decomposition. This is not generally valid in inorganic devices but is thought to hold quite generally in OSCs, where Anderson localization dominates the energy landscape.

Moving forward, a number of extensions of the present work should be explored. Most importantly, to make simulations of this type into a predictive tool, these ideas should be applied to a wider array of OSC systems. Here we demonstrate the techniques on a single material while carefully testing sensitivity to the computational parameters. Similar calculations for the alphabet soup of popular OSC materials - $\mathrm{C}_{60}$, TPD, P3HT, CuPc ... - will lead to a recipe of simulation parameters most accurate for a range of systems. Guidelines of this sort are crucial in addressing technologically relevant structures, such as donor-acceptor interfaces and bulk heterojunctions.

On a technical level, the weakest step in our methodology is clearly the process of MM sampling. We use a single, long MM trajectory to sample the configuration space. Because these materials are glassy, better phase space sampling could be obtained either from Monte Carlo or parallel tempering simulations. These effects would be particularly important for charge transport, where mobilities are dominated by rare trap configurations in the wings of the distribution. ${ }^{8}$ Further, it is not entirely clear that MM provides an adequate picture of the energy landscape. In this respect, it would be interesting to explore the impact of intramolecular reorganization on the exciton bands using a full QM/MM trajectory. Another key area of improvement is the ab initio description of the molecular excited states. Even with the best existing functionals, TDDFT cannot predict exciton energies to a precision better than $0.3-0.4 \mathrm{eV} .{ }^{32,39}$ Clearly this is unacceptable if one wants to have truly black-box tools for describing a wide range of materials.

The methodology presented above can be further extended to explore an even wider range of essential material properties. Here, we have not computed any charge carrier mobilities, but a prediction of this type is within reach by employing our calculated rates in kinetic Monte Carlo 
simulations. ${ }^{8}$ Alternatively, although the present tutorial has focused on parameters governing charge transfer, analogous simulations of exciton transfer could reveal exciton diffusion lengths in disordered materials. All of the above applications would require some enhancement of our existing computational machinery, but would augment our ability to understand and predict the properties of OSCs.

\section{Acknowledgement}

TV acknowledges funding from the DOE (DE-FG02-07ER46474), NSF (CHE-0547877) and a Packard Fellowship. SD thanks Ben Kaduk for assistance with the CDFT coupling code.

\section{Biography}

Seth Difley received a B.S. in chemistry at University of Utah 2004, and is a graduate student at MIT.

Lee-Ping received a B.S. in physics at UC Berkeley 2006, and is a graduate student at MIT.

Sina Yeganeh received a Ph.D. in chemistry at Northwestern University 2008, and is a Postdoctoral Associate at MIT.

Shane Yost received a B.S. in chemistry at UCSB 2008, and is a graduate student at MIT.

Troy Van Voorhis received a Ph.D. in chemistry at UC Berkeley 2001, and is an Associate Professor at MIT.

\section{Supporting Information Available}

Force field parameters and xyz coordinates of snapshots. This material is available free of charge via the Internet at http: / / pubs . acs . org.

\section{References}

1 Pope, M.; Swenberg, C. E. Electronic Processes in Organic Crystals and Polymers; Oxford University Press: New York, 1999. 
2 Coropceanu, V.; Cornil, J.; da Silva Filho, D. A.; Olivier, Y.; Silbey, R.; Bredás, J. L. Charge transport in organic semiconductors. Chem. Rev. 2007, 107, 926-952.

3 Forrest, S. R. The path to ubiquitous and low-cost organic electronic appliances on plastic. Nature 2004, 428, 911-918.

4 Bredás, J. L.; Calbert, J. P.; da Silva, D. A.; Cornil, J. Organic semiconductors: a theoretical characterization of the basic parameters governing charge transport. Proc. Nat. Acad. Sci. 2002, 99, 5804-5809.

5 Deng, W. Q.; Goddard, W. A. Predictions of hole mobilities in oligoacene organic semiconductors from quantum mechanical calculations. J. Phys. Chem. B 2004, 108, 8614-8621.

6 Kilina, S.; Batista, E. R.; Yang, P.; Tretiak, S.; Saxena, A.; Martin, R. L.; Smith, D. L. Electronic structure of self-assembled amorphous polyfluorenes. ACS Nano 2008, 2, 1381-1388.

7 Scholes, G. D.; Harcourt, R. D. Configuration interaction and the theory of electronic factors in energy transfer and molecular exciton interactions. J. Chem. Phys. 1996, 104, 5054-5061.

8 Kwiatkowski, J. J.; Nelson, J.; Li, H.; Bredás, J. L.; Wenzel, W.; Lennartz, C. Simulating charge transport in tris(8-hydroxyquinoline) aluminium (Alq3). Phys. Chem. Chem. Phys. 2008, 10, $1852-1858$.

9 Nayak, P. K.; Periasamy, N. Calculation of electron affinity, ionization potential, transport gap, optical band gap and exciton binding energy of organic solids using solvation model and DFT. Org. Electron. 2009, In Press.

10 Aaqvist, J.; Warshel, A. Simulation of enzyme reactions using valence bond force fields and other hybrid quantum/classical approaches. Chem. Rev. 1993, 93, 2523-2544.

11 Norton, J. E.; Bredás, J. L. Polarization energies in oligoacene semiconductor crystals. J. Am. Chem. Soc. 2008, 130, 12377-12384. 
12 Garbuzov, D. Z.; Bulovic, V.; Burrows, P. E.; Forrest, S. R. Photoluminescence efficiency and absorption of aluminum-tris-quinolate (Alq3) thin films. Chem. Phys. Lett. 1996, 249, 433437.

13 Curioni, A.; Andreoni, W. Computer simulations for organic light-emitting diodes. IBM J. Res. Dev. 2001, 45, 101-113.

14 Difley, S.; Beljonne, D.; Van Voorhis, T. On the singlet-triplet splitting of geminate electronhole pairs in organic semiconductors. J. Am. Chem. Soc 2008, 130, 3420-3427.

15 Marcus, R. A. Theory of oxidation-reduction reactions involving electron transfer. J. Chem. Phys. 1956, 24, 966-978.

16 Karl, N. Charge carrier transport in organic semiconductors. Synthetic Metals 2003, 133, 649_ 657.

$17 \mathrm{Wu}, \mathrm{Q}$.; Van Voorhis, T. Direct calculation of electron transfer parameters through constrained density functional theory. J. Phys. Chem. A 2006, 110, 9212-9218.

18 Van Voorhis, T.; Kowalczyk, T.; Kaduk, B.; Wang, L. P.; Cheng, C. L.; Wu, Q. The diabatic picture of electron transfer, reaction barriers, and molecular dynamics. Ann. Rev. Phys. Chem. 2010, 61, In press.

19 Pacher, T.; Cederbaum, L. S.; Köppel, H. Adiabatic and quasidiabatic states in a gauge theoretical framework. Adv. Chem. Phys. 1993, 84, 293-391.

$20 \mathrm{Wu}, \mathrm{Q}$; Van Voorhis, T. Direct optimization method to study constrained systems within density-functional theory. Phys. Rev. A 2005, 72, 024502.

21 Oberhofer, H.; Blumberger, J. Charge constrained density functional molecular dynamics for simulation of condensed phase electron transfer reactions. J. Chem. Phys. 2009, 131, 064101.

$22 \mathrm{Wu}, \mathrm{Q}$; Van Voorhis, T. Extracting electron transfer coupling elements from constrained density functional theory. J. Chem. Phys. 2006, 125, 164105. 
23 Lamoureux, G.; Roux, B. Modeling induced polarization with classical Drude oscillators: Theory and molecular dynamics simulation algorithm. J. Chem. Phys. 2003, 119, 3025-3039.

24 Hill, I. G.; Kahn, A.; Soos, Z. G.; Pascal, R. A., Jr. Charge-separation energy in films of [pi]conjugated organic molecules. Chem. Phys. Lett. 2000, 327, 181-188.

25 Krause, S.; Casu, M. B.; Scholl, A.; Umbach, E. Determination of transport levels of organic semiconductors by UPS and IPS. New J. Phys. 2008, 10, 085001.

26 Brinkmann, M.; Gadret, G.; Muccini, M.; Taliani, C.; Masciocchi, N.; Sironi, A. Correlation between molecular packing and optical properties in different crystalline polymorphs and amorphous thin films of mer-tris(8-hydroxyquinoline)aluminum(III). J. Am. Chem. Soc. 2000, 122, 5147-5157.

27 Lin, B. C.; Cheng, C. P.; You, Z. Q.; Hsu, C. P. Charge transport properties of tris(8hydroxyquinolinato)aluminum(iii): why it is an electron transporter. J. Amer. Chem. Soc. 2004, $127,66-67$.

28 Hess, B.; Kutzner, C.; van der Spoel, D.; Lindahl, E. GROMACS 4: Algorithms for highly efficient, load-balanced, and scalable molecular simulation. J. Chem. Theory Comput. 2008, 4, $435-447$.

29 Brooks, B. R.; Bruccoleri, R. E.; Olafson, B. D.; States, D. J.; Swaminathan, S.; Karplus, M. CHARMM - a program for macromolecular energy, minimization, and dynamics calculations. J. Comput. Chem. 1983, 4, 187-217.

30 Shao, Y. et al. Advances in methods and algorithms in a modern quantum chemistry program package. Phys. Chem. Chem. Phys. 2006, 8, 3172-3191.

31 Woodcock III, H. L.; Hodošček, M.; Gilbert, A. T. B.; Gill, P. M. W.; Shaefer III, H. F.; Brooks, B. R. Interfacing Q-Chem and CHARMM to perform QM/MM reaction path calculations. J. Comp. Chem. 2007, 28, 1485-1502. 
32 Dreuw, A.; Head-Gordon, M. Single-reference ab initio methods for the calculation of excited states of large molecules. Chem. Rev. 2005, 105, 4009-4037.

33 Djurovich, P. I.; Mayo, E. I.; Forrest, S. R.; Thompson, M. E. Measurement of the lowest unoccupied molecular orbital energies of molecular organic semiconductors. Org. Electron. 2009, 10, 515-520.

34 Schmidt, A.; Anderson, M. L.; Armstrong, N. R. Electronic states of vapor deposited electron and hole transport agents and luminescent materials for light-emitting diodes. J. Appl. Phys. 1995, 78, 5619-5625.

35 Martin, R. L.; Kress, J. D.; Campbell, I. H.; Smith, D. L. Molecular and solid-state properties of tris-(8-hydroxyquinolate)-aluminum. Phys. Rev. B 2000, 61, 15804-15811.

36 Tanaka, I.; Tokito, S. Phosphorescent-sensitized triplet-triplet annihilation in tris 8hydroxyquinoline aluminum. J. Appl. Phys. 2005, 97, 113532.

37 Cölle, M.; Gärditz, C. Phosphorescence of aluminum tris(quinoline-8-olate). Appl. Phys. Lett. 2004, 84, 3160-3162.

38 Burrows, H. D.; Fernandes, M.; de Melo, J. S.; Monkman, A. P.; Navaratnam, S. Characterization of the triplet state of tris(8-hydroxyquinoline)aluminium(iii) in benzene solution. J. Am. Chem. Soc. 2003, 125, 15310-15311.

39 Peach, M. J. G.; Benfield, P.; Helgaker, T.; Tozer, D. J. Excitation energies in density functional theory: An evaluation and a diagnostic test. J. Chem. Phys. 2008, 128, 044118. 


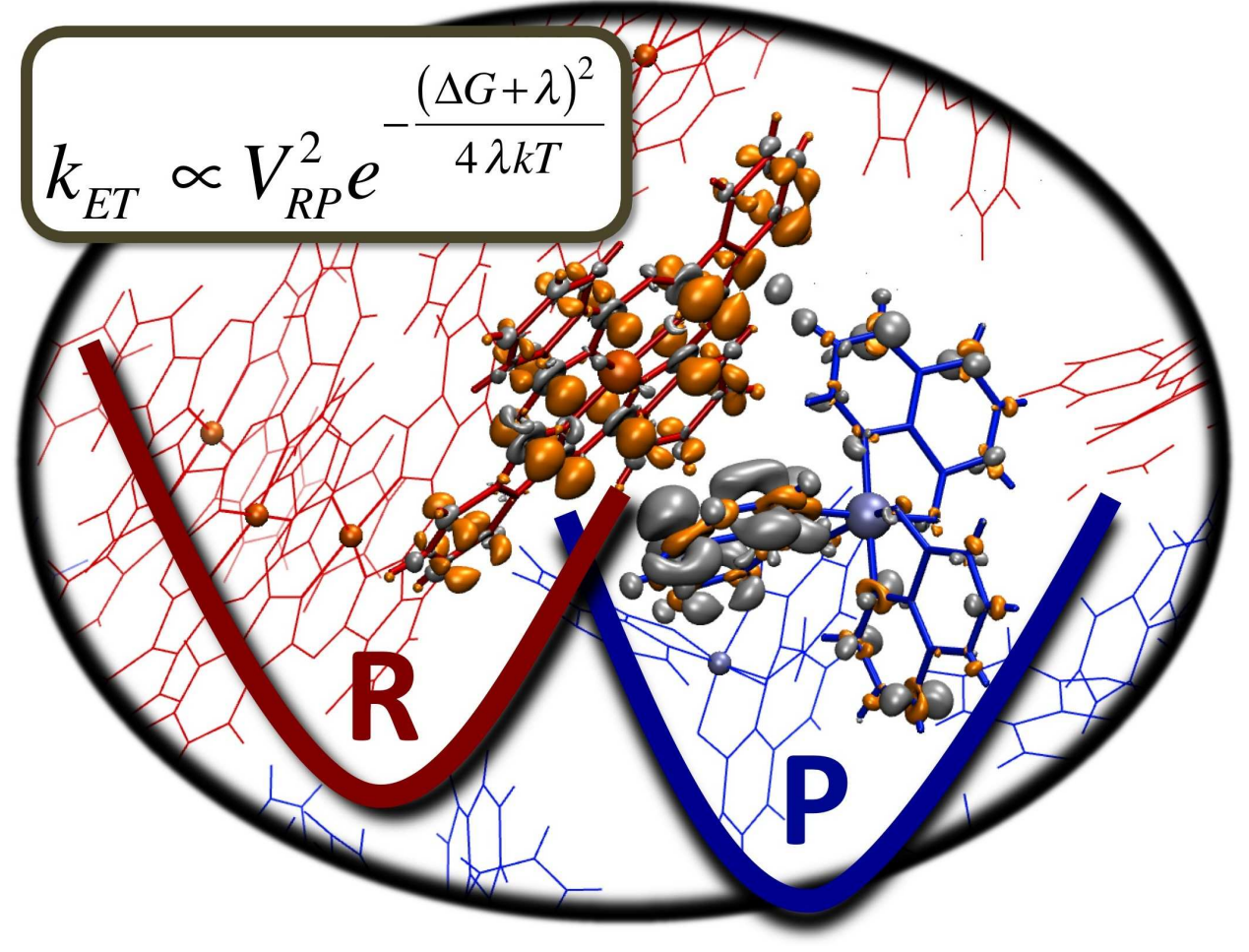

